\title{
Lifetime of a single bubble on the surface of a water and ethanol bath
}

\author{
Elise Lorenceau $~^{1}{ }^{1}$ and Florence Rouyer $\circledast^{2}$,* $^{*}$ \\ ${ }^{1}$ Université Grenoble Alpes, CNRS, LIPhy, F-38000 Grenoble, France \\ ${ }^{2}$ Université Paris-Est, Laboratoire Navier, UMR 8205 CNRS, ENPC ParisTech, IFSTTAR, \\ 5 bd Descartes, 77454 Champs-Sur-Marne, France
}

(Received 7 July 2018; accepted 1 May 2020; published 8 June 2020)

\begin{abstract}
The lifetime of a single bubble on the surface of an alcohol-water liquid bath is measured at intermediate humidity rates. Thanks to an automatic bubble generation device associated with a lifetime measuring device, we carry out a statistical study of the bubble lifetime, which extends over three decades, from 0.1 to $100 \mathrm{~s}$. Up to alcohol concentrations of $12 \%(\mathrm{~g} / \mathrm{g})$, the average bubble lifetime increases with the alcohol concentration. In addition, normalized lifetime probability density functions are independent of the alcohol concentration and relatively well described by a Weibull distribution with an exponent of $4 / 3$. We compare our experimental data with the predictions of a recent model highlighting the importance of Marangoni stresses induced by water contaminants to the fate of a bubble. In our case, these Marangoni stresses are probably induced by heterogeneities in the alcohol concentration maintained by evaporation.
\end{abstract}

DOI: 10.1103/PhysRevFluids.5.063603

\section{INTRODUCTION}

A bubble that bursts at the air/liquid interface typically ejects hundreds of air drops due to the disintegration of the thin hemispherical liquid film separating the interior of the bubble from the atmosphere. This phenomenon has important consequences in volcanology, for example. Since the magmatic processes that govern explosive volcanic eruptions are impossible to observe directly, indirect observations - of the average size or size distribution of the ejected droplets, for exampleprovide a better understanding of the underground magmatic activity [1]. In addition, the droplets ejected when bubbles burst at the sea surface are also a key factor in the study of air-sea interactions. Indeed, they contribute significantly to the evaporation of seawater [2] and are among the main sources of sea salt aerosol. By efficiently scattering solar radiation and serving as nuclei for cloud formation, these aerosols are particularly relevant to understanding radiative forcing of the climate. [3,4].

In this context, the lifetime of a bubble on the surface of a liquid is an important parameter: the longer the lifetime, the more the film is drained and the finer the aerosol formed. Overall, the life of a bubble at an interface is the sum of the characteristic times of drainage, evaporation, hole initiation, and bursting [5-9]. It therefore subtly depends on various parameters such as the size of the bubble, the viscosity of the liquid, and the presence of surfactant molecules [1,5,7,913]. Indeed, by modifying the structure of the interface, the presence of surfactant molecules can increase the lifetime of air bubbles by several orders of magnitude $[5,9,13,14]$. The lifetime of bubbles generated from a liquid consisting of a binary solution can also be very long as observed for saline solutions [15-17], water-alcohol mixtures [7,18,19], oil [20,21], and molten glass [10,22]. For these binary systems, the bubble stabilization mechanism at the liquid/air interface is less well

*florence.rouyer@u-pem.fr 
known. In the case of water/alcohol mixtures, the increase in stability may be due to adsorption of the alcohol at the interface. The high concentration of $-\mathrm{OH}$ dipoles at the air/water interface would induce repulsive van der Waals forces between the interfaces, leading to a disjoining pressure able to balance capillary and gravity drainage of the film $[18,19,23,24]$. This positive adsorption of alcohol has been demonstrated indirectly in experiments on adsorption isotherms and directly in molecular dynamics simulations for methanol and ethanol [25,26]. One could also think of a modification of the surface tension or surface rheology due to the presence of alcohol: Since regions of high surface tension exert higher tangential stresses on the air/liquid interface than regions of low surface tension, a surface tension gradient induces a flow of liquid away from the regions of low surface tension. These Marangoni stresses can even partially counteract gravity or capillary stress, thus stabilizing liquid film [22], single bubbles [27,28], or foam [29]. In this very comprehensive framework, one question remains open: Through which mechanisms common to all these systems are these Marangoni stresses maintained? The answer probably lies in the difference in volatility between the two compounds of the binary system, as recently shown for multicomponent lubricating oils [20]. The overall mechanism can be easily understood within the geometry of a single bubble on the surface of an alcohol/water mixture. The alcohol evaporates everywhere at the same rate. At the foot of the bubble, this evaporation is counteracted by the proximity of the reservoir that replenishes the liquid film, thus ensuring that the temperature and alcohol concentration in the film are equal to those in volume. However, at the top of the bubble, the liquid reservoir is not directly available, which induces a lower alcohol concentration and possibly a lower temperature than in volume. As the surface tension of the alcohol-water mixture decreases with the alcohol concentration or temperature, this creates a surface tension gradient that pulls the liquid upward, partially neutralizing the thinning effects of gravity and capillarity [7,20]. Despite this very convincing mechanism, correlating the importance of these Marangoni flows with bubble life is a delicate task. Indeed, for high-energy surfaces such as water/alcohol solutions, bubble life is the result of both deterministic processes such as gravity, capillarity, and Marangoni stresses and stochastic processes such as the presence of impurities in the air or the solution, which can trigger film rupture [7]. To test the validity of this deterministic/stochastic coupling, it is necessary to work on large assemblies of bubbles and measure their lifetime distribution. This has been done recently for oils [20,21], hot or cold clean water-for which thermal rather than solutal Marangoni flows may appear-and salt solutions [7]. However, the case of alcoholic binary solutions has been less studied [7,18,27]. In particular, the evolution of the bubble lifetime distribution with the alcohol concentration has never been considered.

To fill this gap and to try to quantify the relative importance between stabilization induced by alcoholic adsorption at the interface or by Marangoni flow, we measure the lifetime of individual bubbles on the surface of an ethanol/water liquid bath in an atmosphere of controlled composition. To obtain sufficient statistics, we use a device that allows us to repeat the same experiment more than a hundred times. The average lifetime and bubble lifetime distribution are presented and analyzed for various concentrations of ethanol in the reservoir.

\section{MATERIALS AND METHODS}

\section{A. Materials}

The container is filled with a binary mixture of purified water (conductivity, $18.2 \mathrm{M} \Omega / \mathrm{cm}$ ) and ethanol (Sigma Aldrich; 96\% pure). The ethanol concentration varies from 0 to $12 \%$ (g/g). Within this range of ethanol concentrations, the physical properties of the mixture can vary significantly. Therefore, the density $\rho$, the surface tension $\gamma$, the dynamic viscosity $\eta$, and the diffusion coefficient $D$ of the alcohol-water mixture are listed in Table I.

To form a bubble, ambient air is pumped by an aquarium pump operated by an automated electronic system, then blown through a vertical capillary tube with a diameter of $300 \mu \mathrm{m}$ immersed a few centimeters below the air/liquid interface (see Fig. 1). To ensure proper alignment, the 
TABLE I. Liquid density, liquid/air surface tension, dynamical viscosity, diffusion coefficient, radius of the bubble cap, and number of bubbles for different percentages of ethanol. The liquid density, surface tension, and viscosity are derived from [30], and the diffusion coefficient from [31].

\begin{tabular}{lcccccc}
\hline \hline $\begin{array}{l}\% \text { ethanol } \\
(\mathrm{g} / \mathrm{g})\end{array}$ & $\begin{array}{c}\rho \\
(\mathrm{kg} / \mathrm{L})\end{array}$ & $\begin{array}{c}\gamma \\
(\mathrm{mN} / \mathrm{m})\end{array}$ & $\begin{array}{c}\eta \\
(\mathrm{mPa} \mathrm{s})\end{array}$ & $\begin{array}{c}D \\
\left(\mathrm{~m}^{2} / \mathrm{s}\right)\end{array}$ & $\begin{array}{c}R \\
(\mathrm{~mm})\end{array}$ & \begin{tabular}{c}
$N_{\text {bubbles }}$ \\
\hline 0
\end{tabular} \\
\hline 1 & 0.997 & 71.1 & 0.89 & $1.22 \times 10^{-9}$ & - & 250 \\
2.5 & 0.996 & 65.9 & 0.92 & $1.19 \times 10^{-9}$ & 3.5 & 1250 \\
5 & 0.993 & 60.9 & 0.97 & $1.14 \times 10^{-9}$ & 3.3 & 1000 \\
7 & 0.989 & 56.1 & 1.04 & $1.07 \times 10^{-9}$ & 3.1 & 250 \\
10 & 0.986 & 52.7 & 1.11 & $1.02 \times 10^{-9}$ & 3.0 & 250 \\
12 & 0.986 & 49.1 & 1.21 & $0.94 \times 10^{-9}$ & 3.0 & 500 \\
\hline \hline
\end{tabular}

capillary tube is placed along the axis of symmetry of the $4.5-\mathrm{cm}$-wide cylindrical vessel. Once detached from the cylindrical vessel, the bubble rises up and stabilizes at the center of the air/liquid interface. Indeed, the surface is slightly convex thanks to a hydrophobic Teflon ring placed at the top of the container, which imposes a contact angle greater than $90^{\circ}$ along the walls. Unless otherwise specified, the experiments are performed at $24^{\circ} \mathrm{C} \pm 2{ }^{\circ} \mathrm{C}$ in an atmosphere with an ethanol partial pressure and a water partial pressure of $1365 \pm 45 \mathrm{~Pa}$, corresponding to a humidity level of $45.5 \% \pm 1.5 \%$ (measured with a TESTO 625 probe).

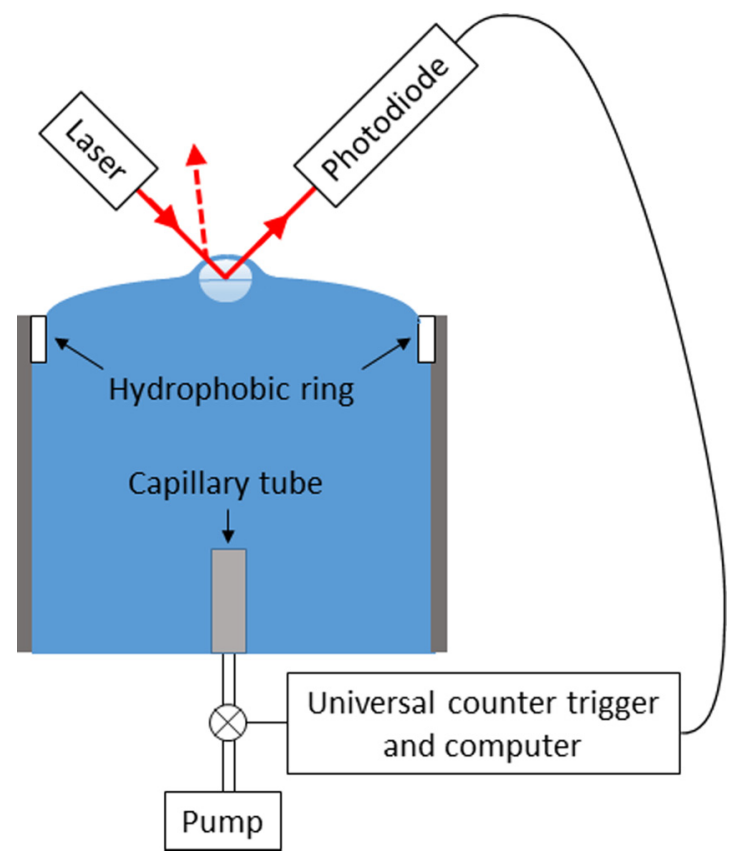

FIG. 1. Setup used to form bubbles and to measure their lifetimes on the surface of the liquid bath. The dashed red arrow corresponds to the reflection of the laser beam when a bubble is at the interface, while the solid red arrow corresponds to the reflection of the laser beam when there is no bubble at the interface. 
TABLE II. Capillary length $l_{c}$, viscous time $\tau$, and Schmidt (Sc) and Bond (Bo) numbers calculated from the values in Table I for different percentages of ethanol.

\begin{tabular}{lcccc}
\hline \hline $\begin{array}{l}\% \text { ethanol } \\
(\mathrm{g} / \mathrm{g})\end{array}$ & $\begin{array}{c}l_{c} \\
(\mathrm{~mm})\end{array}$ & $\begin{array}{c}\tau \\
\left(10^{-6} \mathrm{~s}\right)\end{array}$ & Bo & Sc \\
\hline 0 & 2.70 & 33.8 & 1.68 & 735 \\
1 & 2.60 & 36.3 & 1.82 & 779 \\
2.5 & 2.50 & 39.7 & 1.79 & 850 \\
5 & 2.40 & 44.7 & 1.65 & 982 \\
7 & 2.33 & 49.1 & 1.65 & 1102 \\
10 & 2.25 & 55.6 & 1.76 & 1306 \\
12 & 2.20 & 60.5 & 1.62 & 1459 \\
\hline \hline
\end{tabular}

\section{B. Automatic bubble generation and lifetime measurement}

Bubble lifetime on the surface of the liquid bath is measured by means of a laser beam inclined approximately $45^{\circ}$ and directed towards the center of the cylindrical container. If the liquid/air interface is flat and undistorted, the laser beam is reflected by the liquid interface and its intensity is recorded using a photodiode (see Fig. 1). If the liquid/air interface is distorted by the presence of a bubble, then the laser reflection is deflected and the photodiode does not record any light intensity. Once the bubble has burst, the liquid/air interface returns to its equilibrium position and the laser beam is reflected back to the photodiode, which stops recording the time. Thus, the lifetime of a bubble corresponds to the time interval during which there is no signal at the output of the photodiode. It is measured using a universal counter triggered by the photodiode signal switch. Five seconds after the bubble burst, the universal counter triggers the formation of a new bubble and the process is repeated. Measured in this way, the typical lifetime of a bubble $t$ is determined with an accuracy of $\pm 0.2 \mathrm{~s}$, corresponding to the damping of the oscillations induced by the bursting of the bubble. It typically varies from a few milliseconds to $100 \mathrm{~s}$.

The radius at the apex of the bubbles obtained with this setup varies from 2.8 to $3.5 \mathrm{~mm}$. As the shape of the bubble results from an equilibrium between gravity and capillary forces [32], we introduce the capillary length $l_{c}=\sqrt{\gamma /(\rho g)}$, which varies from 2.6 to $2.2 \mathrm{~mm}$. The dimensionless parameter $R / l_{c} \approx 1.31 \pm 0.04$ as reported in Table II. This corresponds to a Bond number Bo $=$ $R^{2} / l_{c}^{2}=\rho g R^{2} / \gamma \sim 1.6$ of the order of unity as reported in Tables I and II. It is reproducible within $5 \%$ tolerance due to slight variations in the ethanol concentration induced by evaporation.

This device can therefore generate a large number of data as long as the external experimental conditions (such as the liquid bath level, ambient atmosphere, and temperature or humidity level) do not change. The number of bubbles generated for each alcohol concentration, listed in Table I, varies from 250 to 1250 .

\section{RESULTS AND DISCUSSION}

Figure 2 presents typical raw data on the bubble life in a $12 \%(\mathrm{~g} / \mathrm{g})$ ethanol-water bath. For this assembly of 340 bubbles, the lifetime varies over three decades, from 0.1 to $100 \mathrm{~s}$. To quantify these data, we successively consider the average bubble lifetime, $t_{b}$, as well as the probability density function (PDF) of observing a bubble with a given lifetime.

\section{A. Average lifetime}

First, we check that the average lifetime of the bubble $t_{b}$ does not change over time. To do this, we average over dozens of bubbles rather than over the entire range: plotted against the number of bubbles, the values obtained do not show any significant variation. Then we calculate $t_{b}$ over the entire range. For the data presented in Fig. $2, t_{b}=29.2 \pm 4.9 \mathrm{~s}$. 


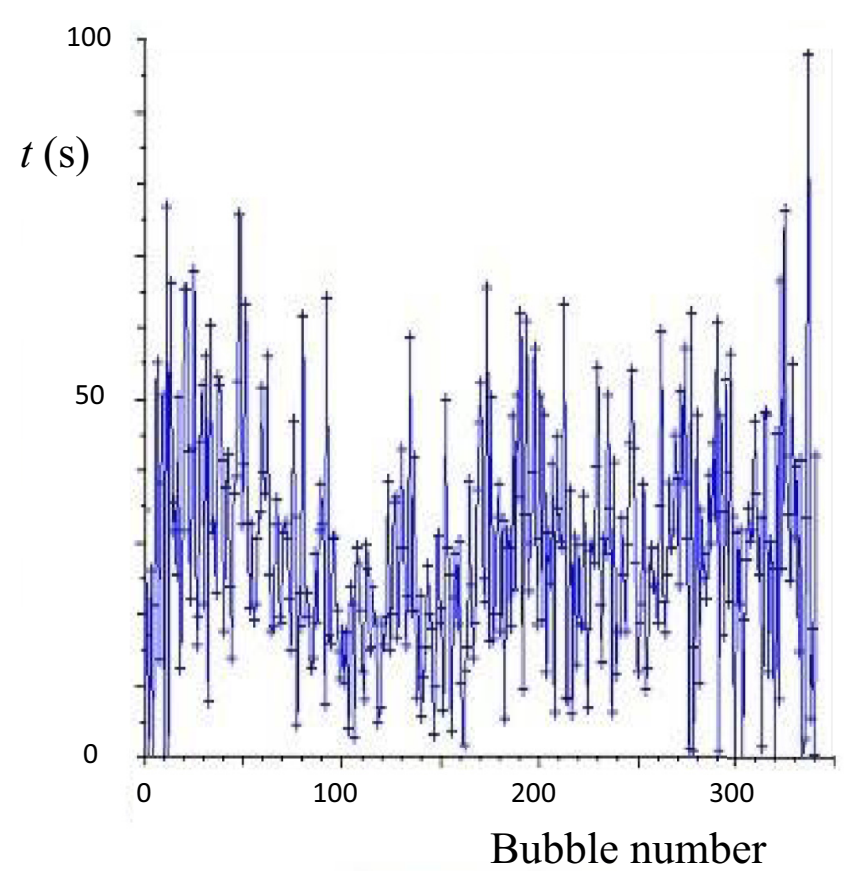

FIG. 2. Bubble lifetime, $t$, as a function of the bubble number for $12 \%(\mathrm{~g} / \mathrm{g})$ alcohol in water.

The average life of the bubbles, plotted as a function of the ethanol concentration in Fig. 3, increases with the concentration of ethanol and is well described by a linear (empirical) adjustment passing through 0 . This is not surprising: at $0 \%$ ethanol, the film of pure water at the top of the bubble

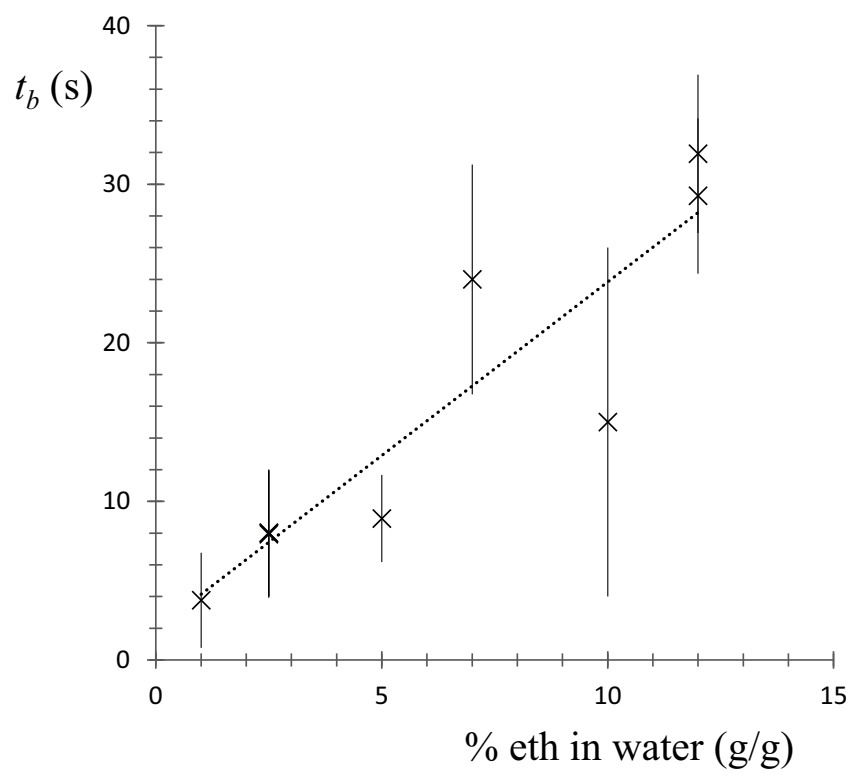

FIG. 3. Average bubble lifetime, $t_{b}$, as a function of the percentage $(\mathrm{g} / \mathrm{g})$ ethanol in water. The error bar corresponds to the standard deviation. The linear fit is a guide for the eyes. 
being in no way stabilized, the bubble explodes in a few milliseconds, which is much too short a duration for our experimental automatic device and negligible compared to the average lifetime measured at higher ethanol concentrations. We were unable to work at an ethanol concentration greater than 12\%: above this value, the contact angle of the binary mixture on the vertical wall is smaller, thus reducing the amplitude of the meniscus and its ability to stabilize the bubble in the center of the tank. We also carry out the same experiment but in the presence of a vapor saturated with ethanol and water. In the absence of evaporation, the bursting is quasi-immediate and the response time in our setup (of the order of $0.2 \mathrm{~s}$ ) is too large to quantitatively probe this effect. All these results are consistent with a stabilization mechanism of bubbles induced by Marangoni effects and by the evaporation of ethanol as presented in Sec. I. Indeed, if the vapor is not saturated with ethanol, evaporation generates concentration heterogeneities at the bubble scale. As the surface tension of water/ethanol mixtures decreases with increasing ethanol concentration, these concentration heterogeneities generate surface tension gradients that can drag the liquid upwards. These Marangoni stresses can even counteract the gravitational and capillary thinning of the film, making it less likely to explode. However, if the vapor is saturated with ethanol, the ethanol cannot evaporate and the bubble bursts rapidly.

This stabilizing effect observed over a range of ethanol concentrations from 0 to $12 \%(\mathrm{~g} / \mathrm{g})$ is also observed in [29]. However, while we observe increasing stability with ethanol concentration up to $12 \%(\mathrm{~g} / \mathrm{g})$, Tuinier et al. observe maximum foamability for a concentration of $5 \%-7 \%(\mathrm{~g} / \mathrm{g})$ ethanol in water. However, comparison between the two experimental works cannot be made easily. In Tuinier and coworkers' experiment, the most fragile bubbles, located at the top of a foam column, are far away from the ethanol tank. Moreover, as the system is closed, unlike here, it is possible that the vapor quickly saturates with ethanol, thus reducing evaporation. The two main ingredients of the stabilization mechanism we describe in this paper, namely, evaporation and ethanol concentration gradients, are therefore probably limited in the geometry of the Tuinier et al. experiment.

\section{B. Probability density function (PDF) of the bubble lifetime}

In Fig. 4, the PDFs for the minimum and maximum ethanol concentrations are plotted on a lin-lin scale. The PDF of a random positive variable $x$ is often described using a Weibull distribution, $p(x)=\beta / x_{0}\left(x / x_{0}\right)^{\beta-1} \exp \left(-\left(x / x_{0}\right)^{\beta}\right)$, where $\beta>0$ is the shape parameter and $x_{0}>0$ is the scale parameter. In the case where $x$ is a value to failure, the failure rate and $\beta$ are related: $\beta=1$ corresponds to a constant failure rate for which the distribution is a monotonously decreasing exponential function, while $\beta=2$ corresponds to an increasing failure rate for which the distribution is a Rayleigh distribution, also often used to describe statistics of a positive random variable based on diffusion, $p(t)=\pi / 2\left(t / t_{b}^{2}\right) \exp \left(-\pi / 4\left(t / t_{b}\right)^{2}\right)$. In the problem considered here, the bursting of the bubbles is triggered by the appearance of thinner areas of the film. By carefully monitoring the rate of film thinning and assuming that the PDF of the bubble lifetime is related to the frequency of appearance of these independent fragile parts, Lhuissier showed that the distribution of the lifetime of unpurified water bubbles is well described by a Weibull distribution with $\beta=4 / 3$, given by

$$
q(t)=\frac{4}{3}\left(\frac{t^{1 / 3}}{t_{0}^{4 / 3}}\right) \exp \left(-\left(\frac{t}{t_{0}}\right)^{4 / 3}\right),
$$

where $t_{0}$ is proportional to $t_{b}, t_{0}=t_{b} / \Gamma(1+3 / 4) \sim t_{b} / 0.92$, with $\Gamma$ the gamma function [33]. And, indeed, we observe a reasonable agreement between Eq. (1) and the two sets of data in Fig 4.

In Fig. 5, the normalized PDFs obtained for all ethanol concentrations are plotted on a lin/log scale as a function of $t / t_{b}$, using for $t_{b}$ the previously determined average lifetime. We observe a collapse of the experimental PDFs. Yet for $t / t_{b}>3$, the data are very scattered. However, for this normalized time range, the number of data is low. To qualify the importance of the least statistically significant data, two types of symbols of the same color are used for each alcohol concentration. Filled symbols are data for which more than 10 events are counted and open symbols are those 


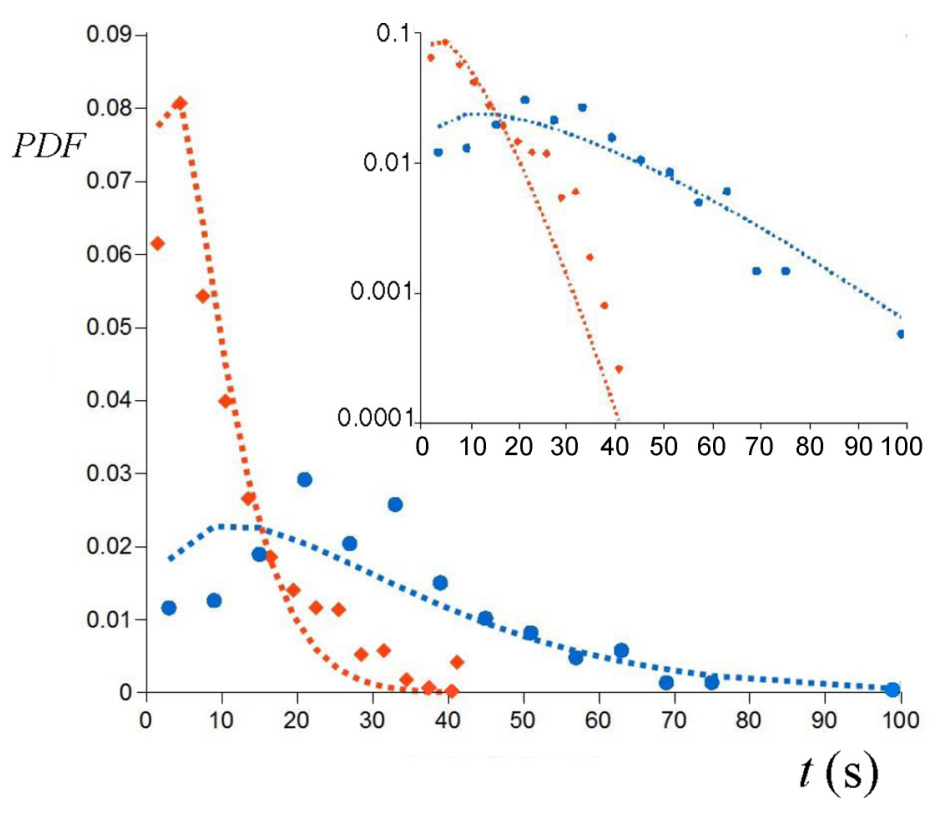

FIG. 4. Probability density function (PDF) of experimental bubble lifetimes for $2.5 \%$ (red diamonds; over 1251 bubbles) and 12\% (blue circles; over 312 bubbles) alcohol in water $(\mathrm{g} / \mathrm{g})$. Dotted lines are Weibull distributions [5,33], Eq. (1). Inset: Same data plotted on log-lin axis.

for which fewer than 10 events are counted. For all ethanol concentrations, we lack statistics as soon as $t / t_{b}>3$. These normalized experimental PDFs are now compared with the Weibull PDFs described previously. First, the exponential function (i.e., the Weibull distribution with $\beta=1$ ) which corresponds to random external events causing a break and constant over time does not allow us to capture the increase in probability at low lifetimes. We also compare in Fig. 5 normalized PDFs along with Rayleigh distributions as done in [20] and [34]. A Rayleigh distribution (i.e., Weibull distribution with $\beta=2$ ) also fits our data correctly for $t / t_{b}<2$ but seems to fail to catch the tail of the distribution. Finally, a good agreement with Weibull's law with $\beta=4 / 3$ [see Eq. (1)] is observed without any other adjustable parameter. This fit is particularly good up to $t / t_{b}<3$, which is precisely the range of data for which we have a sufficient statistic. In addition, since PDFs seem to be independent of the alcohol concentration, we merge all the data in the same graph (see inset in Fig. 5). This increases the statistics for the tail of the distribution and confirms that the longterm distribution is better described by a Weibull function than by a Rayleigh function. Our results therefore suggest that the function's distribution of the bubble lifetime is more stretched than the Rayleigh function based solely on diffusive processes and narrower than the exponential function based solely on the time-independent failure rate.

\section{Discussion}

During the life of a bubble, liquid film thins inexorably until bursting. This thinning dynamics can be controlled either by the evaporation rate of the liquid (water being the main component of these films) or by the drainage of the liquid due to various mechanical stresses (such as gravity or capillary stresses).

It is presumably the composition of the liquid constituting the films and of the atmosphere as well as the bubble size that favor one or the other of the scenarios. For example, films of water bubbles at temperatures from $15^{\circ} \mathrm{C}$ to $77^{\circ} \mathrm{C}$ in unsaturated atmospheres-and thus subject to evaporation-are always thicker than those of bubbles in saturated atmospheres. This counterintuitive observation 


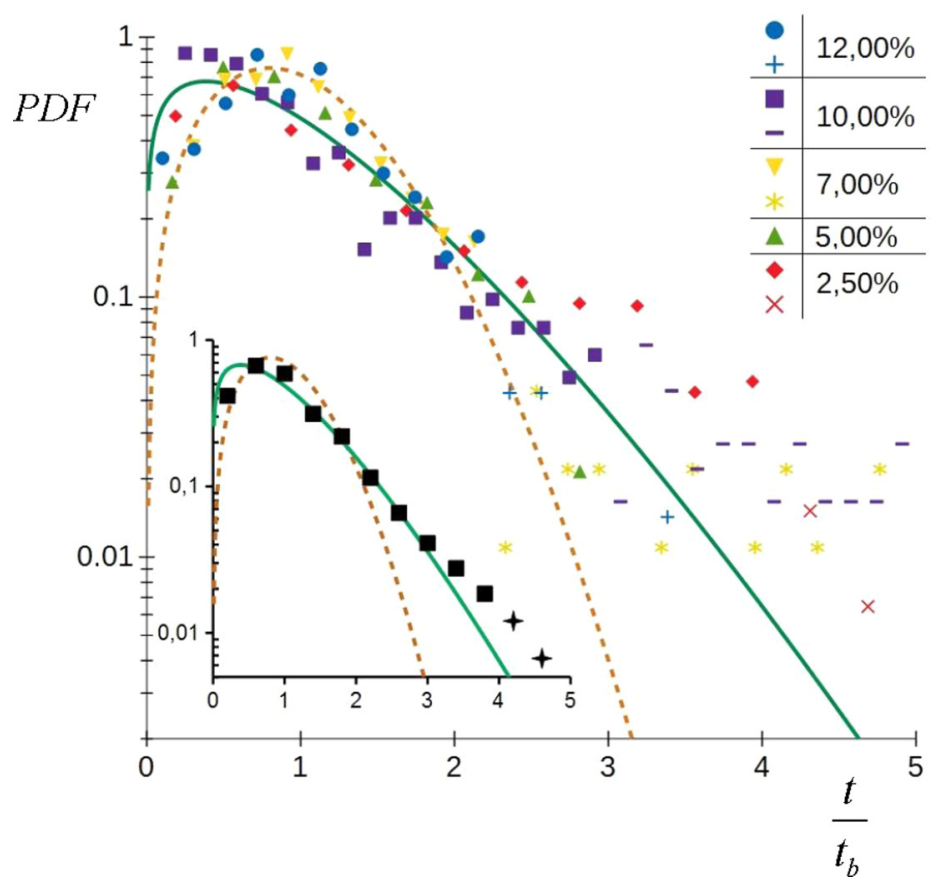

FIG. 5. Probability density function (PDF) of bubble lifetimes normalized by average lifetimes for five concentrations of ethanol in water, varying from $2.5 \%$ to $12 \%(\mathrm{~g} / \mathrm{g})$ (see legend). Two types of symbols of the same color are used for each concentration. Filled symbols are data for which more than 10 events are counted and open symbols are those for which fewer than 10 events are counted. The solid line represents the Weibull function [5,33], and the dashed line the Rayleigh function. Inset: PDF for all normalized lifetimes- $t / t_{b}-$ whatever the ethanol concentration.

shows that for this system the mass loss induced by evaporation is negligible on the time scale of the bubble life [7]. On the contrary, films of bubbles stabilized by amphiphilic molecules adsorbed at the film interface are barely subject to drainage since, in this case, Marangoni stresses balance the gravitational or capillary stresses. This induces much longer lifetimes, for which mass loss induced by evaporation is no longer negligible [8,9]. Using the framework developed in [8] and [9], assuming that this is the liquid evaporation that limits the maximum lifetime accessible to very stable bubbles [i.e., evaporation of a film of critical thickness for which both film thinning phenomena induced by capillary drainage (lower and lower over time) and evaporation are of the same order (constant over time)], one can estimate the bubble lifetime $t_{b}^{\text {evap }} \approx R\left(\left(\frac{\rho}{J}\right)^{3}\left(\frac{\eta R}{\gamma l_{c}}\right)^{2}\right)^{1 / 5}$, where $J$ is the liquid mass evaporation rate.

Taking pure liquid ethanol evaporation rate values for ranges of intermediate humidity [35], as well as the bubble size $R$, surface tension $\gamma$, viscosity $\eta$, and liquid density $\rho$ as listed in Table I, we find $t_{b}^{\text {evap }}$ at a maximum of $10 \mathrm{~s}$ of the same order of magnitude as our observations. Yet, this value decreases $20 \%$ as the ethanol concentration increases from $1 \%$ to $12 \%(\mathrm{~g} / \mathrm{g})$. Film thinning induced only by evaporation is therefore in disagreement with our data.

Thus, for the bubbles we are considering, film thinning is likely to be controlled by liquid drainage. In this case, the addition of ethanol to the aqueous solution constituting the film can modify this drainage dynamic. Indeed, ethanol can adsorb at the interface and modify various surface parameters such as the surface viscosity, which would slow down the drainage of the film [36]. It can also induce solutal or thermal Marangoni stresses that would cause the liquid in the bath to rise up, thus slowing down the thinning rate due to capillary or gravity stresses [7]. To stabilize 
the bubble for a long enough time, these stresses must be sustained either by evaporation or by a temperature difference between the atmosphere and the bulk.

Stabilization induced by adsorption of surface active agents should not be sensitive to the composition of the vapor phase. However, when the experiment is carried out in an atmosphere saturated with alcohol-which should allow a maximum concentration of molecules adsorbed on the surface - the stabilizing effect disappears and all the bubbles burst in less than a second. This mechanism is therefore probably not dominant here, and in the rest of this paper, we assume that the thinning dynamics of films is governed by the generalized drainage model proposed in [5] and [7].

To go one step further and understand what governs the death of the bubbles, we now recall the different pictures proposed in the literature. A classic deterministic scenario suggests that films thin out until they are thin enough for van der Waals forces or temperature fluctuations to break down. However, at the time of rupture, experimental measurements of film thicknesses are found to be highly dispersed, ranging from a few nanometers to a micrometer, suggesting a stochastic scenario. Recently, a very comprehensive framework has been proposed to understand what governs the death of a bubble $[5,7,9]$. Stochastic local perturbations arising from intrinsic perturbations such as impurities from the solution or extrinsic ones such as impacts by mist droplets are responsible for the bubble's death. The authors show that the ability of these perturbations to kill the bubble are not all equivalent: they are highly efficient bubble killers upon reaching the interface at the bubble foot and when coupled with contaminants inducing Marangoni stresses large enough to trigger self-sustained lethal thinning.

Thus, by coupling a deterministic generalized bubble cap drainage model accounting for both curvature-pressure-induced drainage and Marangoni flows and the time scales involved in the sideto-side diffusion with the time of residence of the stochastic contaminant on the bubble, the authors of [5] and [7] show (i) that the adequacy between the measured PDF and a Weibull distribution is theoretically justified and (ii) that the bubble lifetime reads as

$$
t_{b}=\alpha \tau \operatorname{Sc}\left(\frac{R}{l_{c}}\right)^{1 / 2}
$$

where $\tau=\frac{\eta l_{c}}{\gamma}$ is the viscocapillary time and Sc the Schmidt number, Sc $=\frac{\eta}{\rho D}$, with $D$ the diffusion coefficient of the impurities in the aqueous solution. In the following we assume that this diffusion coefficient is equal to the mutual diffusion of the ethanol-water mixture (cf. Table I) because in the present case a local variation of the ethanol concentration would affect the surface tension of ethanol-water, similarly to impurities at the surface of the water. Typical values of $\tau$ and Sc are listed in Table I. The prefactor $\alpha$ is a numerical coefficient accounting for the efficiency of Marangoni stresses to counteract gravitocapillary drainage, which reads

$$
\alpha=\frac{1}{1 / 3-k \mathrm{Bo}}
$$

where $k$ is a numerical coefficient accounting for the reduction in the thinning rate of the films due to Marangoni stresses. For a hot film in cold air, $k$ has been found to be of the order of its maximal value, $k \sim 0.05<1 / 3 \mathrm{Bo}$, whatever the value of the temperature difference [7] for Bo $\sim 4$.

To determine whether water/ethanol thin film stability can be described using this picture, we plot $t_{b}$ as a function of $\tau \operatorname{Sc}\left(R / l_{c}\right)^{1 / 2}$ in Fig. 6. Although Bo and $\rho$ do not vary significantly, $\gamma, \eta, D$, and thus $\tau$ and Sc (whose values are listed in Tables I and II) all vary significantly with the ethanol contents in the probed range. The data in Fig. 6 are reasonably well described by a linear variation with a slope $\alpha$ of the order of 350. Note that a plot of $t_{b}$ versus $\tau$ shows a strong nonlinear variation; instead, $t_{b} \propto \tau^{3.5}$. The difference between the linear law observed between $t_{b}$ and $\tau \operatorname{Sc}\left(R / l_{c}\right)^{1 / 2}$ and a power law between $t_{b}$ and $\tau$ with an exponent greater than 3 may seem surprising. However, we recall that $D \propto 1 / \eta$, thus $\operatorname{Sc} \propto \eta^{2}$ and $\tau \operatorname{Sc}\left(R / l_{c}\right)^{1 / 2} \propto \eta^{3} \sim \tau^{n}$, with $n \sim 3$. Using Eqs. (2) and (3) as well as the values of Bo in Table II yields a constant value of $k \mathrm{Bo} \approx 0.3305 \approx<1 / 3$, which is 


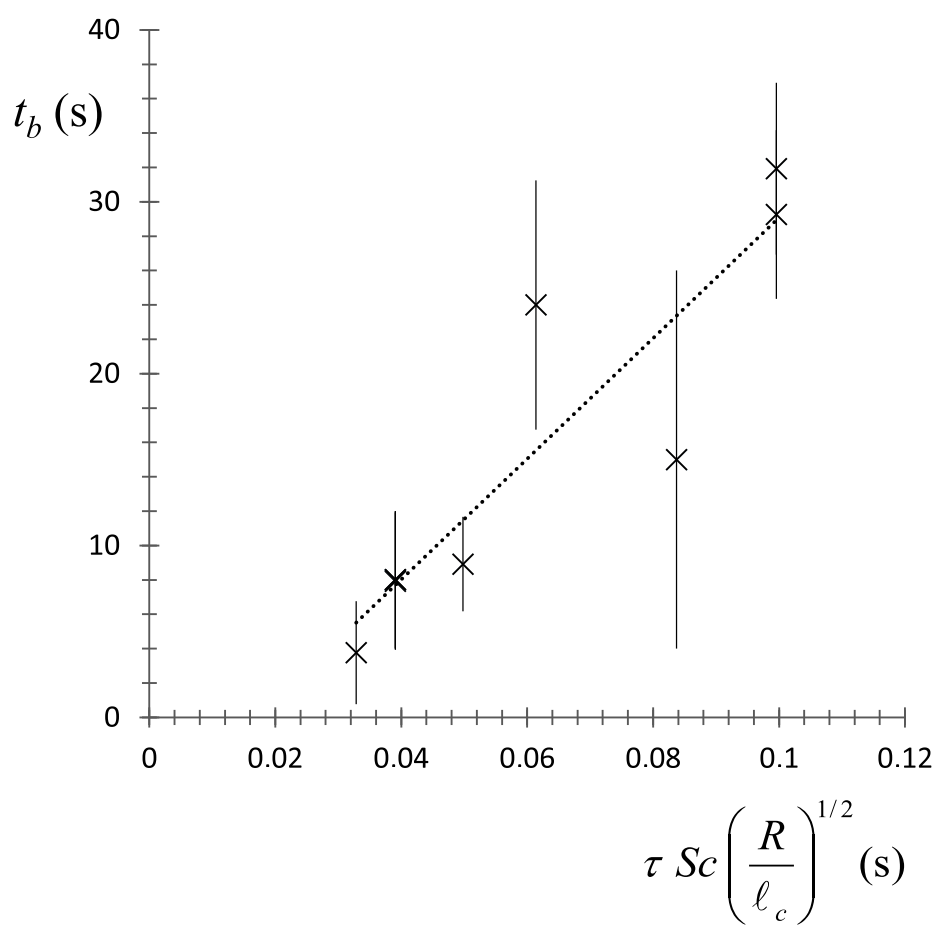

FIG. 6. Average bubble lifetime as a function of $\tau \operatorname{Sc}\left(\frac{R}{l_{c}}\right)^{1 / 2}$.

consistent with the maximal value predicted in [7] and $k \approx 0.2$. Our work thus reaffirms that even if eventually undergoing stochastic rupture, the lifetime of bubbles stabilized by upward Marangoni stresses (solutal due to the evaporation of ethanol in this case) rather than by surfactants can be modeled by an equilibrium between the diffusion time and the residence time of the contaminant at the foot of the bubble. The film at the foot of the bubble is very thin and subject to violent evaporation of ethanol in the present case. This very particular geometric configuration (confined film and evaporation) could be responsible for the stochastic appearance of large local concentration gradients.

The normalized PDF shown in the inset in Fig. 5 also agrees very well with the prediction from [5] and [7]. Indeed, when the bubble lifetime is normalized by the mean bubble lifetime, all the data are observed to collapse on a master curve perfectly fitted by Eq. (4). A small deviation occurs for the data with the longest lifetime $\left(t / t_{b}>4\right)$, which are a bit overrepresented. This deviation remains unexplained.

\section{CONCLUSION}

A statistical study of thousands of bubbles shows that the average lifetime of bubbles on the surface of an ethanol-water bath increases considerably with the concentration of ethanol. Our data are consistent with the framework in [5] and [7]: This increase can be understood by considering the ethanol concentration heterogeneities induced by evaporation, which in turn create upward Marangoni stresses. In order to validate this picture, several hypotheses remain to be validated, notably that significant ethanol concentration gradients may appear on the bottom of the bubble and that the stochastic appearance of these gradients are due to the evaporation of the very thin film of liquid. In addition, several questions remain unanswered, in particular, those concerning the 
thickening of the film on top of the bubble due to the evaporation of the surface-active species as observed on vertical films [10,20,37] and on the surface of the bubbles [7].

\section{ACKNOWLEDGMENTS}

We acknowledge the technical team of Laboratoire Navier for realizing the setup. We thank P. Willay and D. Dessalles for conducting experiments. We thank E. Rio, J. Miguet, Q. Magdelaine, A. Antkowiak, O. Atasi, and B. Scheid for fruitful and stimulating discussions. We gratefully acknowledge financial support from IFSTTAR and ENPC for internship remuneration.

[1] C. T. Nguyen, H. M. Gonnermann, Y. Chen, C. Huber, A. A. Maiorano, A. Gouldstone, and J. Dufek, Film drainage and lifetime of bubbles, Geochem. Geophys. Geosyst. 14, 3616 (2013).

[2] E. L. Andreas, J. B. Edson, E. C. Monahan, M. P. Rouault, and S. D. Smith, The spray contribution to net evaporation from the sea: A review of recent progress, Bound.-Layer Meteorol. 1, 3 (1995).

[3] D. M. Murphy, J. R. Anderson, P. K. Quinn, L. M. McInnes, F. J. Brechtel, S. M. Kreidenweis, A. M. Middlebrook, M. Posfai, D. S. Thomson, and P. R. Buseck, Influence of sea-salt on aerosol radiative properties in the Southern Ocean marine boundary layer, Nature 392, 62 (1998).

[4] S. E. Schwartz, The Whitehouse effect-Shortwave radiative forcing of climate by anthropogenic aerosols: An overview, J. Aerosol Sci. 27, 359 (1996).

[5] H. Lhuissier and E. Villermaux, Bursting bubble aerosols, J. Fluid Mech. 696, 5 (2012).

[6] E. Rio and A.-L. Biance, Thermodynamic and mechanical timescales involved in foam film rupture and liquid foam coalescence, ChemPhysChem 15, 3692 (2014).

[7] S. Poulain, E. Villermaux, and L. Bourouiba, Ageing and burst of surface bubbles, J. Fluid Mech. 851, 636 (2018).

[8] J. Miguet, M. Pasquet, F. Rouyer, Y. Fang, and E. Rio, Stability of big surface bubbles: Impact of evaporation and bubbles sizes, Soft Matter 16, 1082 (2019).

[9] S. Poulain and L. Bourouiba, Biosurfactants Change the Thinning of Contaminated Bubbles at BacteriaLaden Water Interfaces, Phys. Rev. Lett. 121, 204502 (2018).

[10] H. Kočárková, F. Rouyer, and F. Pigeonneau, Film drainage of viscous liquid on top of bare bubble: Influence of the Bond number, Phys. Fluids 25, 022105 (2013).

[11] G. Debregeas, P. G. de Gennes, and F. Brochard-Wyart, The life and death of bare viscous bubbles, Science 279, 1704 (1998).

[12] R. L. Modini, L. M. Russell, G. B. Deane, and M. D. Stokes, Effect of soluble surfactant on bubble persistence and bubble-produced aerosol particles, J. Geophys. Res. Atmos. 118, 1388 (2013).

[13] L. Champougny, M. Roché, W. Drenckhan, and E. Rio, Life and death of not so bare bubbles, Soft Matter 12, 5276 (2012).

[14] S. Ross and G. Nlshloka, Foaminess of binary and ternary solutions sydney, J. Phys. Chem. 79, 1561 (1975).

[15] J. J. Bikerman, Persistence of bubbles on inorganic salt solutions, J. Appl. Chem. 18, 266 (1968).

[16] S. R. Burger and D. C. Blanchard, The persistence of air bubbles at a seawater surface, J. Geophys. Res.: Oceans 88, 7724 (1983).

[17] M. Struthwolf and D. C. Blanchard, The residence time of air bubbles less than $400 \mu \mathrm{m}$ diameter at the surface of distilled water and seawater, Tellus Ser. B. Chem. Phys. Meteorol. 36, 294 (1984).

[18] S. Samanta and P. Ghosh, Coalescence of air bubbles in aqueous solutions of alcohols and nonionic surfactants, Chem. Eng. Sci. 66, 4824 (2011).

[19] O. Oolman, Timothy and W. Blanch, Harvey, Bubble coalescence in stragnant liquids, Chem. Eng. Commun. 43, 237 (1986).

[20] V. Chandran Suja, A. Kar, W. Cates, S. M. Remmert, P. D. Savage, and G. G. Fuller, Evaporation-induced foam stabilization in lubricating oils, Proc. Natl. Acad. Sci. USA 115, 7919 (2018). 
[21] M. Arangalage, J.-P. Gingras, N. Passade-Boupat, F. Lequeux, and L. Talini, Asphaltenes at oil/gas interfaces: Foamability even with no significant surface activity, Colloids Interf. 3, 2 (2019).

[22] F. Pigeonneau, H. Kočárková, and F. Rouyer, Stability of vertical films of molten glass due to evaporation, Colloids Surf. A: Physicochem. Eng. Aspects 408, 8 (2012).

[23] G. Drogaris and P. Weiland, Coalescence behavior of gas bubbles in aqueous solutions of $\mathrm{n}$-alcohols and fatty acids, Chem. Eng. Sci. 38, 1501 (1983).

[24] L. Wang and R.-H. Yoon, Effects of surface forces and film elasticity on foam stability, Int. J. Miner. Process. 85, 101 (2008).

[25] J. J Kipling, Composition of the surface film on aqueous solutions of aliphatic alcohols, J. Colloid Sci. 18, 502 (1963).

[26] M. A. Wilson and A. Pohorille, Adsorption and solvation of ethanol at the water liquid-vapor interface: A molecular dynamics study, J. Phys. Chem. B 101, 3130 (1997).

[27] E. Ghabache, Surface libre hors équilibre : de l'effondrement de cavité aux jets étirés, Ph.D. thesis, Université Pierre et Marie Curie, 2015.

[28] V. V. Yaminsky, Bubble vortex at surfaces of evaporating liquids, J. Colloid Interf. Sci. 297, 251 (2006).

[29] R. Tuinier, C. G. J. Bisperinl, C. Van Denberg, and A. Prins, Transient foaming behavior of aqueous alcohol solutions as related to their dilational surface properties, J. Colloid Interf. Sci. 179, 327 (1996).

[30] I. S. Khattab, F. Bandarkar, M. A. A. Fakhree, and A. Jouyban, Density, viscosity, and surface tension of water plus ethanol mixtures from 293 to 323K, Korean J. Chem. Eng. 29, 812 (2012).

[31] K. R. Harris, T. Goscinska, and H. N. Lam, Mutual diffusion-coefficients for the systems water-ethanol and water propan-1-op at $25^{\circ} \mathrm{C}$, J. Chem. Soc. Faraday Trans. 89, 1969 (1993).

[32] H. M Princen, Shape of a fluid drop at a liquid-liquid interface, J. Colloid Sci. 18, 178 (1963).

[33] T. Gilet, T. Scheller, E. Reyssat, N. Vandewalle, and S. Dorbolo, How long a bubble will be, arXiv:0709.4412 [physics.flu-dyn]..

[34] Q. A. Zheng, V. Klemas, and Y.-H. L. Hsu, Laboratory measurements of water surface bubble life time, J. Geophys. Res. 88, 701 (1983).

[35] P. L. Spedding, J. Grimshaw, and K. D. O'Hare, Abnormal evaporation rate of ethanol from low concentration aqueous solutions, Langmuir 9, 1408 (1993).

[36] J. Seiwert, R. Kervil, S. Nou, and I. Cantat, Velocity Field in a Vertical Foam Film, Phys. Rev. Lett. 118, 048001 (2017).

[37] J. B. Fournier and A. M. Cazabat, Tears of wine, Europhys. Lett. (EPL) 20, 517 (1992). 